The section of less than 5000 words to be read is the main paper without sect. 4 and Appendix.

This is a preprint of a paper forthcoming in Erkenntnis whose final and definitive version is available here: $\underline{\text { http://link.springer.com/article/10.1007/s10670-015-9777-3 }}$

\title{
A Situationalist Solution to the Ship of Theseus Puzzle
}

\section{Martin Pickup}

\author{
Abstract This paper outlines a novel solution to the Ship of Theseus puzzle. The \\ solution relies on situations, a philosophical tool used in natural language semantics among \\ other places. The core idea is that what is true is always relative to the situation under \\ consideration.
}

I begin by outlining the problem before briefly introducing situations. I then present the solution: in smaller situations (containing only one of the candidate ships for identity with Theseus's ship) the candidate is identical to Theseus's ship. But in larger situations containing both candidates these identities are neither true nor false. Finally, I discuss some worries for the view that arise from the nature of identity, and suggest responses. It is concluded that the solution, and the theory that underpins it, are worth further investigation. 
The aim of this paper is to suggest a new solution to the Ship of Theseus puzzle, which I call situationalist. The solution is, with minor adjustments, also applicable to the variety of other puzzles about the continued existence of material objects over time. ${ }^{1}$ I focus here on the Ship of Theseus puzzle to give space to flesh out the account more fully.

I proceed by first outlining the problem we are faced with by the Ship of Theseus. I will then in the second section briefly introduce the theoretical machinery that I need to articulate the novel solution. This is a particular characterisation of situations, which are philosophical tools used elsewhere (especially in semantics and information theory) and can be broadly thought of as parts of possible worlds. By settling on a view of the relations between situations, we open us space for the new solution. This follows in section 3 . Section 4 considers some objections and sketches replies, which then leaves us in a position to conclude.

Before embarking on our task, though, we ought to pause. There are a suite of available solutions to the puzzles of persistence, so one might wonder what adding to their number achieves. This speculation gains further force for my particular presentation of the situationalist solution, for I shall not argue that it is the correct solution but merely an interesting one that warrants further investigation. This is, to be frank, because I myself am not yet completely convinced the costs of the solution outweigh its benefits. And there is a yet further reason to worry about the value of the solution: it resembles other existing solutions. ${ }^{2}$ So we might think it is otiose: if it is a restatement or a mere refinement this significantly undermines its appeal.

Thus there is a need to make explicit the motivations for this paper. These correspond to a primary and a subsidiary aim. The primary aim is to present the virtues of the situationalist 
solution. In the first place, the solution is not, I argue, a restatement or mere refinement of existing solutions. Although it does conclude that certain identity statements are indeterminate in certain situations, and that these statements are determinate in others, these statements are capable of varying in truth-value even across different situations in the same world at the same time. ${ }^{3}$ As another point of contrast, for situationalism the indeterminacy of the identity statements is due to indeterminacy in the world, not in representation or language. ${ }^{4}$ In addition, it embeds this claim in a wider picture that sees the indeterminacy of these identity statements as instances of a more general phenomenon. The solution offered is, in fact, a broader theory that solves the puzzles of persistence as a by-product. Furthermore, as I will substantiate in section 3 , it allows us to hold onto the relevant intuitions in each set of circumstances in ways that similar solutions do not, including giving an account for why the Ship of Theseus case is puzzling. It also gives a distinctive and natural response to the criticisms levelled against solutions which use indeterminate identity, which is described in section $4 .{ }^{5}$ Finally, as mentioned above, the mechanics of the solution are applicable to a wider range of other problems (including the problem of change). This univocal means of solving a wide range of problems is a major benefit and highlights the difference between this solution and the others it resembles, which are not so widely applicable.

The subsidiary aim of the paper is more general. I believe there is a benefit to framing the discussion in terms of situations - the use of situations to describe the Ship of Theseus case gives us a neat and succinct way to formalise the issues at stake. The particular solution I describe arises from a certain way of understanding the relations between situations, but even if one isn't sympathetic to the situationalist solution the clarity that the use of situations brings seems worthy of dissemination. 
For these two reasons, then, I take it that the situationalist picture is worth having on the table. Now we can begin the process of describing it.

\section{The Ship of Theseus Puzzle}

The Ship of Theseus puzzle is one that comes down to us from Ancient Greece, via Plutarch and Hobbes. ${ }^{6}$ It can be set up as follows: imagine there is a ship arriving in Athens harbour. This is Theseus' ship, returning from Crete and the defeat of the Minotaur. Call this ship 'Victory'. The Athenians, overjoyed at the safe return of Theseus and the young people of Athens, decide to put Victory on display in a museum. As the months and years go by, parts of the ship lose their lustre and are worn down. These worn planks are replaced by new ones when necessary by the curator of the museum. After 20 years, all of the planks of Victory have been replaced. Call the ship that is in the museum after 20 years 'Restoration'. Is Restoration Victory? The natural answer is that it is; ships can survive the gradual replacement of parts.

Now imagine that, instead of displaying Victory when it arrives back in Athens, the Athenians take it apart and leave it in a warehouse. After 20 years, someone suggests they put the ship back together for a commemorative celebration of Theseus' return. So, they put the planks back together and display the ship on the Acropolis for their party. Call the ship on the Acropolis 'Reconstruction'. Is Reconstruction Victory? Again, the natural answer is that it is; ships can survive being taken apart and put back together.

But here comes the puzzle. Suppose that the Athenians do decide to display the ship in the museum. And suppose, further, that the curator stores all the replaced planks in a warehouse. On the 20th anniversary of Theseus' return, these planks are put back together 
on the Acropolis. Where is Victory? Is it in the museum? Or is it on the Acropolis? Or neither? Or both? In other words, which, if either, of the following are true: Restoration = Victory; Reconstruction = Victory?

The difficulty that the Ship of Theseus case presents is that none of the answers to this question seems satisfactory. When we are considering Restoration in isolation it seems like it is identical to Victory. Likewise when we just attend to Reconstruction. But when both Restoration and Reconstruction are in the picture we don't know what to say. There is a desire to maintain the identities, but Victory cannot be identical to both Restoration and Reconstruction (for they are not identical to one another and identity is transitive). But how can what we do with the replaced planks affect whether or not Restoration is identical to Victory? And, similarly, how can what happens while the original planks are removed affect whether or not Reconstruction is identical to Victory? So we have a tension between the wish to maintain the identities in the first two stories but not in the third. In the third scenario it seems the identities are simply unclear. ${ }^{7}$ I will claim that situations can resolve this tension and capture the different intuitions the three stories elicit.

To be clear then, I take the following to be the claims we wish to maintain: (i) in the first story, Victory is identical to Restoration, (ii) in the second story, Victory is identical to Reconstruction, (iii) in the third story, Victory is not obviously identical to either, but nor is it obviously distinct from either. What we are looking for, therefore, is a philosophically respectable way to uphold all of these claims.

\section{Situations}


In this section, we will give a brief account of situations to prepare the ground for the new solution which follows.

\subsection{Introduction to Situations}

Situations are a philosophical tool which have been used in a couple of different areas. The most important role they play is in natural language semantics and, relatedly, in information theory. To get a grip on what situations are, it is helpful heuristic to first think of them as parts of possible worlds. Examples include the part of the world containing (only) the battle of Hastings, the part of the world containing (only) the first human footstep on Mars and the part of the world containing (only) the behaviour of subatomic particles on the head of a particular pin.

As I am understanding them, situations are concrete portions of the world. They are also, by their nature, typically partial: the situation that contains only a certain cat being tabby does not include the other properties of that cat. Situations are restricted in their purview. The actual world can then be seen as a special sort of situation that is in some sense maximal. So far we have described only actual situations. But I take there to be merely possible situations too; situations which could but do not obtain. Possible worlds, consequently, are situations which are maximal as the actual world situation is maximal. One natural way to understand this is that they cover all of a region of space and time that is causally connected or connectible. ${ }^{8}$ So in our framework possible worlds are in fact special sorts of situation and thus situations can be seen as prior to possible worlds. ${ }^{9}$

We are still not precisely clear what situations are though. Kratzer, when using them in situation semantics, identifies them with Armstrong's states of affairs. ${ }^{10}$ For Kratzer, then, a situation is an instantiation by a thin particular of a universal. But Armstrong's is a 
contentious ontology, and I prefer to steer clear of the controversies it can engender. I will simply stipulate the following: situations are composed of particulars' having of relations (including properties as unary relations). More precisely, actual situations are composed of actual particulars' having of actual relations. Merely possible situations are what would be composed by possible particulars' having of possible relations. ${ }^{11}$ If Armstrong is right about the ontology, this will collapse into the view presented by Kratzer, but I do not want to be committed to it. So this is what situations are, then: particulars' standing in relations or having properties. ${ }^{12}$

\subsection{Two key notions: truth in a situation and parthood}

We now need to introduce two key notions in situation theory. The first is that of truth in a situation. We might say that a proposition is true with respect to a situation iff the proposition accurately expresses the way things are in that certain portion of a world. ${ }^{13}$ This is one of the distinctive features of situation theory: propositions are never true simpliciter, but only true with respect to some situation. ${ }^{14}$ We can therefore describe propositions as true in situations when the contents of the situation correspond to what the proposition says. ${ }^{15}$ As a slogan, we can then say: all truth is truth in a situation. It is worth giving some examples to show more fully how the proposal works. So; consider a cat, called Puddy, who is a tabby. The proposition expressed by 'Puddy is tabby' is true in situations containing Puddy being tabby. But the proposition is not true in a situation that doesn't contain Puddy being tabby. The proposition that everybody is happy is true in situations in which every person is, in fact, happy. The proposition that at least a dozen people support Arsenal is true in situations in which 12 or more people support Arsenal. In general, situation semantics permits propositions to be true in some situations but not others. 
But how does this notion of truth in a situation work? We can draw on an analogy with possible worlds. We are familiar with the notion of a propositions being true in worlds; expressions of the type, 'proposition $p$ is true in world $w$ '. How this is interpreted obviously depends on your general view of truth (and indeed of worlds), but roughly stated it means that things are as $p$ says they are in world $w$. This rough statement is what we can use for truth in a situation too: $p$ is true in a situation $s$ when things are as $p$ says they are in $s$. Cashing this out in more detail will depend on your other commitments. ${ }^{16}$

A final point is worth making at this stage about truth in a situation. What happens when a proposition is about particulars or relations that are not in the relevant situation? For example, if we consider the situation which only contains Puddy being tabby, what is the status in this situation of the proposition 'Puddy is furry'? The proposition is not true in that situation: the situation is not as the proposition says it is, for the property 'furry' is not in the situation. But this leaves us two options. It could be false in the situation, or it could be neither true nor false in the situation. I prefer the latter alternative. This has a few consequences. The first is that the operator 'true in s' isn't bivalent: it doesn't return either true or false for every proposition. The second is that, on an intuitive understanding of 'or', the operator doesn't obey the law of excluded middle. It is not always the case that the proposition that either $p$ or not- $p$ is true in $s$ because there are some situations in which both $p$ and not- $p$ are neither true nor false. ${ }^{17}$ The third consequence is that there is space and need for an account of what it is for a proposition to be false in a situation. We take falsity in a situation to be a primitive, much as truth in a situation is.

There are technical questions arising from the brief sketch of truth in a situation above. Some will find it easier to understand the proposal if it is spelt out formally. For these readers, it is worth looking at the Appendix, where a semantics is given that gives more of 
an outline of the machinery underlying the rest of the paper. But, of course, some others will find it easier to understand the proposal without the formal material. For this reason, and for the flow of the paper, the technical aspects are consigned to the Appendix.

This gives us a grip on what it is for a proposition to be true or false in a situation. Now we can turn to one of the key foundational concepts in situation theory, namely parthood. Situations can be parts of one another. Take the situation of what is happening in a particular house during a particular 24 hour period. This situation, call it $s$, will have other situations as parts. For example, the situation $s^{\prime}$, which is what happens during that 24 hour period in the kitchen, will be part of $s$. Likewise, $s^{\prime \prime}$, which is what happens during one hour of that 24 hour period in the whole house, will also be a part of $s$. And, of course, $s$ itself will be a proper part of larger situations and an improper part of itself.

Parthood is the key relation between situations. ${ }^{18}$ It is transitive, reflexive and antisymmetric. Parthood for situations is often defined as follows: $s^{\prime} \leq s$ iff $s$ contains all the particulars in $s^{\prime}$, instantiating all the properties and relations they do in $s^{\prime}$. If ' + ' denotes a simple sum function, this can be expressed as $s^{\prime} \leq s$ iff $s+s^{\prime}=s .^{19}$

\subsection{Transference}

This introductory material gives us the tools to express the central contention of the paper. Is a proposition true in a part of a situation always true in the whole situation? Consider two situations, $s$ and $s^{\prime}$, where $s^{\prime} \leq s$. And consider a proposition $p$ which is true in $s^{\prime}$. Is $p$ thereby required to be true in $s$ ? Unhelpfully, this question is termed in the literature a question about the persistence of propositions across situations. The terminology is obviously liable for confusion. I will therefore propose the term transfer. A proposition 
transfers between $s^{\prime}$ and its extension $s$ iff it is true in both $s^{\prime}$ and $s$. The issue under consideration here is whether all propositions transfer.

Proponents of situations are divided on this question. Kratzer (1989) accepts universal transference (at least for propositions expressed in natural language). Elbourne (2005) and Barwise and Perry (1983) deny it. It is key to our project that universal transference doesn't hold. It seems a prima facie plausible principle, however, so we need to motivate such a denial.

There are some problem cases for the claim that all propositions transfer. One such case is universal generalisation. Consider the proposition, 'all trees are laden with wonderful apples' (Kratzer's example). This proposition might be true in some situation $s^{\prime}$ containing only a well-appointed orchard. But consider another situation $s$ which has $s^{\prime}$ as a part; say the situation containing the well-appointed orchard and the surrounding countryside for 100 miles. The proposition is not true in this situation: $s$ contains trees which are not laden with wonderful apples. Similar cases emerge when we consider certain other quantifiers, as well as superlatives and the definite article. ${ }^{20}$ These together seem to undermine the universality of transference.

There are methods by which we can save universal transference here. Kratzer, for instance, posits implicit quantifier domain restriction in cases of universal quantification, and this might be extended to the other examples. But, as well as complicating the formal semantics, this seems at odds with the project of situation semantics. Situation semantics is designed to account for the fact that the very same proposition can be true and false in different parts of the same world; it is in virtue of being more fine-grained than possible world semantics that it claims an advantage. Implicit quantifier domain restriction, on the other hand, is a way of asserting that two utterances which appear to express the same 
proposition in fact express different ones. I take it to be preferable for situation semantics to hold onto the claim that the very same proposition is expressed in $s^{\prime}$ and $s$, but that the truth-value of the proposition varies.

There is much more to be said in this debate, some of it formal and some philosophical. At the very least, though, the denial of universal transference seems one coherent way to understand propositions, situations and their relations. I will deny universal transference. I hold that, in some circumstances, a proposition true in a part of a situation is not true in the whole situation. I hope that the use I find for this might also contribute to a case for such a denial.

Although I claim that transference is not universal, of course in most cases propositions do transfer. The fact that most propositions do transfer needs to be accounted for. I propose a restricted transference principle. This principle will govern when propositions must transfer and when they might not. The crux of the principle is that propositions have to transfer unless what the proposition is talking about changes. In other words, propositions always transfer when the larger situation says nothing more about the referents of the proposition. But it might not when the larger situation does say something about the referents of the proposition. To refine this we need to introduce a technical notion, that of an existential situation. An existential situation for some particular $a$ is simply a situation in which $a$ exists. So propositions transfer in all cases except those where additional existential situations for relevant particulars are in the larger situation; in such a case the proposition may fail to transfer. Thus:

Restricted Transference: if $p$ is true in $s^{\prime}$ and $s^{\prime} \leq s$ then $p$ is true in $s$, unless $s$ contains more existential situations for particulars that $p$ picks out than $s^{\prime}$. 
The upshot of this principle is that if $p$ refers to things that are more replete in $s$ than $s^{\prime}$ then $p$ might not transfer. It might be that $p$ is true in $s^{\prime}$ without being true in $s$. It also might be the case the $p$ is true in $s^{\prime}$, but this is not guaranteed by the structural relations. In other words, the principle describes a sufficient but not necessary condition for transference.

An example may make the role of restricted transference clearer. The example of universal quantification above was: 'all trees are laden with wonderful apples'. This is true in $s^{\prime}$. But it fails to transfer to $s$, which has $s^{\prime}$ as a part. Why is this? Because $s$ contains at least one further tree which is not laden with wonderful apples. Restricted transference captures this, because trees are particulars picked out by the proposition, and there are extra existential situations for trees in $s$ that are not in $s^{\prime}$. So the proposition fails restricted transference and isn't required to be true in $s$. Indeed, the proposition is not true in $s$, and in virtue of these extra existential situations (i.e. the extra trees).

In the next section I will demonstrate what the restriction of transference can achieve in providing a solution to the above case of the Ship of Theseus. I call the background theory situationalism and the solution thereby is situationalist. ${ }^{21}$

\section{The Solution to the Ship of Theseus Puzzle}

The outline of the Ship of Theseus case from section 1 needs to be put into the language of situations before our own distinctive solution becomes apparent. So, let's begin by describing some situations: 
$S_{1}$ : The situation containing a ship arriving in port and the removal of this ship to a museum where the planks are gradually replaced until, after 20 years, none remain. $S_{2}$ : The situation containing a ship arriving in port and the history of the planks of this ship, being gradually moved to a warehouse until they are reformed into a ship on the Acropolis after 20 years.

$S_{3}$ : The situation containing a ship arriving in port, the removal of this ship to a museum where the planks are gradually replaced until, after 20 years, none remain and the history of the planks of the initial ship, being gradually moved to a warehouse until they are reformed into a ship on the Acropolis after 20 years.

$S_{3}$ has $S_{1}$ and $S_{2}$ as parts but $S_{1}$ and $S_{2}$ are not parts of each other. The situations we have given describe what happens in parts of the world. The problematic situation is $S_{3}$, which contains all the others. Let's see how the problem arises by looking at some of the propositions true in situations $S_{1}$ and $S_{2}$ :

In $S_{1}:$ Victory $=$ Restoration

In $S_{2}:$ Victory $=$ Reconstruction

So far, so good. But the trouble is that $S_{1}$ and $S_{2}$ are parts of $S_{3}$. So if these propositions transfer between $S_{1}$ and $S_{3}$ and between $S_{2}$ and $S_{3}$ we would have the following:

In $S_{3}$ : Victory = Restoration

Victory $=$ Reconstruction 
But via the transitivity of identity, from Victory $=$ Restoration and Victory $=$ Reconstruction we should add:

$$
\text { Restoration }=\text { Reconstruction }
$$

However, it clearly isn't the case that Restoration = Reconstruction in $S_{3}$; they are distinct ships with different properties and which are located in different places.

Our solution is to deny that these propositions transfer. Rather, in $S_{3}$ neither the proposition 'Victory $=$ Restoration' nor the proposition 'Victory $=$ Reconstruction' are true. We are not entitled to assert either that Victory $=$ Restoration or that Victory $=$ Reconstruction in $S_{3}$ because of our principle of restricted transference. That principle states that propositions may not transfer from a sub-situation to a situation if there are additional existential situations for a particular referred to by the proposition in the larger situation. In $S_{3}$ there are indeed additional existential situations for Victory that are not in $S_{1}$ (and parallel reasoning applies in the case of $S_{2}$ ). For $S_{3}$ contains a situation in which Victory exists which is not in $S_{1}$, namely $S_{2}$. $S_{2}$ is an existential situation for Victory that is not part of $S_{1}$. Thus we have created space for a view where some propositions do not transfer, and this is just such a case where they don't.

We do, however, now have to ask what the status of these propositions is in $S_{3}$. I hold that they are not false, but rather are neither true nor false. ${ }^{22}$ The situationalist account of what is going on can therefore be summarised: in the smaller situations containing only one (good) candidate for identity with Victory the relevant identity claim holds, but in larger situations composed of these smaller situations and in which there is more than one (good) candidate for identity with Victory the identities do not hold. Instead, they are neither true 
nor false. (Note that the situationalist approach works within a world, so even in the same world there can be variation in the truth-values of identities across situations.) I take it that this vindicates the intuitions we have when confronted by the Ship of Theseus case, and spelt out above. For the situationalist is able to claim: (i) Victory is indeed identical to Restoration when considered in isolation, (ii) Victory is indeed identical to Reconstruction when considered in isolation and (iii) in the larger scenario Victory is not obviously identical to either, but nor is it obviously distinct from either. The last point seems to fit our intuitive confusion about what is the case in $S_{3}$. For, when confronted by the whole situation $S_{3}$, it seems we do not know what to say is going on.

I should be explicit here that the situational approach does not force one to hold that the identity of Victory with Restoration and with Reconstruction is indeterminate. It is open to us to adopt a difference stance, for instance claiming that one of the identities holds but the other does not. I prefer to treat Restoration and Reconstruction symmetrically because I have no fixed intuitions about which is Victory in $S_{3} \cdot{ }^{23}$ But the overall machinery is compatible with different assignments of truth-values. In cases of perfectly symmetric fission we would certainly want both propositions to lack a truth-value, but the theory has the flexibility to accommodate different views on asymmetric cases. Nevertheless, I take the challenge of the Ship of Theseus case to be to provide a way of explaining the difficult cases when two (or more) identities seem individually plausible but are jointly incompatible. Situationalism per se doesn't tell us how to assign truth-values, but it instead does allow us to assign them in ways that otherwise appear inconsistent. For this reason I take situationalism to count as a solution to the problem: because the semantics and metaphysics it described allows us to assign truth-values in ways that otherwise lead to inconsistency. 
On encountering the situationalist approach, one might be tempted to ask: what's really the case? Is it what is the case in $S_{1}$ ? Or $S_{2}$ ? Or $S_{3}$ ? The answer is simple: the question is badly formed. What is really the case, or what is the case simpliciter, is not part of the theory. The theory rather states that what is the case is always relative to a situation. There are deep metaphysical questions about the relative primitiveness of different situations: are the larger or the smaller ones more fundamental? I avoid these here. For full disclosure: I take it that smaller situations are more basic. But we do not have space to investigate the metaphysics or ontology, and the theoretical machinery will be compatible with different ways of cashing these out. ${ }^{24}$

So by chopping the world up into situations, we are able to offer a fine-grained answer to the puzzle. By distinguishing between different situations and giving these situations an appropriate structure we can say what we want to say without contradiction.

\section{Objections and replies}

Now that we have outlined the theory, we should outline and respond to some criticisms that naturally arise. The most pressing worries have to do with identity. These are, firstly, that identities are necessary, secondly, that our picture violates Leibniz's Law and thirdly, that identity must be intrinsic.

\subsection{The Necessity of Identity}

The claim that identity is necessary is controversial but widely supported. Barcan and Kripke provided arguments that purported to show that if $a=b$ then necessarily $a=b .{ }^{25}$ I don't have space to fully enter into this debate here. But, if identity is necessary, it might 
seem as though our situationalist solution is in trouble. After all, Victory $=$ Restoration in $S_{1}$ but not in $S_{3}$. This makes it appear contingent whether Victory is identical to Restoration or not.

There are two lines of reply that I want to briefly sketch. In the first instance, what it means to say that a proposition is necessary on a situational account needs to be clarified. We have options: a proposition $p$ could be necessary if it is true in all situations. But is 'all bachelors are unmarried' true in situations containing no bachelors? Or is 'water is $\mathrm{H}_{2} \mathrm{O}$ ' true in situations in which there is no water? This goes against the spirit of situations as partial (with the exception of maximal situations: worlds). One alternative definition of necessity states that $p$ is necessary if it is not false in any situation. This definition is the one I prefer. ${ }^{26}$ On this definition, identity is necessary on the situational account: if $a=b$ in any situation then there is no situation in which it is false that $a=b$. The Ship of Theseus case does not undermine this because our solution doesn't give examples of situations in which the relevant propositions expressing identities are false, but merely that these propositions lack truth-value. ${ }^{27,28}$

Perhaps this seems too easy a response, and the critic will insist that the notion of necessity I employ for situations isn't real necessity. This brings us to the second line of reply to the argument. For in order to evaluate this response, we would need an interpretation of real necessity. This would have to be such that both the situationalist solution violates the real necessity of identity and that the real necessity of identity is provable by the Barcan/Kripke argument. The Barcan/Kripke argument relies on the necessity of ' $x=x^{\prime}$, for any $x$. So we need a definition of necessity that warrants the necessity of ' $x=x^{\prime}$ (for any $x$ ) but not of our identity statements in the situationalist solution. Truth in all situations, as noted above, is problematically restrictive as a definition of necessity for other reasons. But 
it also fails this test: in a situation not containing $a$, even ' $a=a$ ' will plausibly be indeterminate (and hence not true). ' $a=a$ ' is thus not necessary by this definition, and this scuppers the Barcan/Kripke argument.

Consider a second alternative: $p$ is necessary if not false or indeterminate in any situation. But this suffers from the same problem: 'all bachelors are unmarried' is plausibly indeterminate in situations containing no bachelors, given the situational account, and is therefore judged contingent. This is not the right result. More damagingly still, ' $a=a$ ' is plausibly indeterminate in situations not containing a and thus is not necessary. It cannot therefore be used as a premise in the Barcan/Kripke argument for the necessity of identity. ${ }^{29}$

Of course, I have not exhaustively surveyed possible accounts of necessity. So there may be one that both allows the Barcan/Kripke argument and that fails for our identity statements in the situational solution. But we need to be provided with a notion of 'real' necessity that satisfies both of these. Pending such a notion, and an argument for why 'real' necessity is required over and above the interpretation of necessity I've given, I consider the criticism at the least incomplete.

\subsection{Leibniz's Law}

The second challenge claims that the proposed solution violates Leibniz's Law. The thought is the following: if Victory = Restoration is true in $S_{1}$ then they share all properties in common (as an instance of Leibniz's Law). But Victory has some properties Restoration doesn't, like being identical to Reconstruction in $S_{2}$. Thus Victory and Restoration violate Leibniz's Law but the theory claims they are identical. 
To unpick this challenge, we need to be precise about what is being claimed. Victory $=$ Restoration in $S_{1}$. It is part of the theory that truth is situation-relative. So Leibniz's Law should be treated as a schema: for all situations, if $a=b$ then they share all properties. ${ }^{30}$ Hence Victory and Restoration must, by Leibniz's Law, share all properties in common in $S_{1}$. The question is then whether it is true that in $S_{1}$ Victory has the complex property isidentical-to-Reconstruction-in- $S_{2}$, thereby entailing by Leibniz's Law that Restoration have it too. I contend that Victory does not have this property in $S_{1}$. As we made clear when introducing situations, they are partial. For example, Puddy does not have the property of being furry in a situation which contains only Puddy being tabby. Similarly, the complex property is-identical-to-Reconstruction-in- $S_{2}$ refers to something outside the situation $S_{1}$ and hence is not a property that Victory has in $S_{1}$. Neither Victory nor Restoration has it in $S_{1}$ so they do not fail Leibniz's Law there. ${ }^{31,32}$

There is a further worry that arises from Leibniz's Law, however. It can be drawn from an argument against indeterminate identity proposed by Evans (1978) and also by Salmon (1981). The issue concerns what is the case in $S_{3}$. The argument, in our terms, can be put as follows: in $S_{3}$ it is neither true nor false that Victory $=$ Restoration (this is asserted above to save the necessity of identity). Thus it is indeterminate that Victory $=$ Restoration in $S_{3}$. So it seems that in $S_{3}$ Victory has the property of being indeterminately identical to Restoration. However, in $S_{3}$ it is also the case that Restoration $=$ Restoration. So Restoration is identical to Restoration in $S_{3}$ and this seems to entail that it doesn't have the property of being indeterminately identical to Restoration in $S_{3}$.

Thus it seems that Restoration and Victory differ in properties in $S_{3}$. But Leibniz's Law appears to militate against this. Understood as the indiscernibility of identicals, the Law can be expressed as follows: $\forall x \forall y \forall F[[x=y] \rightarrow[F x \rightarrow F y]] .{ }^{33}$ The contrapositive of this is the 
distinctness of discernibles, namely the claim that if two things have different properties then the negation of their identity is true $(\forall x \forall y \forall F[\neg[F x \rightarrow F y] \rightarrow \neg[x=y]])$. So it seems that if Victory and Restoration have different properties in $S_{3}$ then they are distinct. Though this does not formally contradict our previous assertion that it is indeterminate whether Restoration is identical to Victory in $S_{3}$ it is a serious worry. ${ }^{34}$ Evans's argument seems to undermine the claim that identity statements can be neither true nor false, and this in turn would mean that Victory $=$ Restoration would be false in $S_{3}$ and do away with even the weakened sense of the necessity of identity in a situationalist framework. ${ }^{35}$

Luckily, however, we can respond to this worry. The argument requires a particular interpretation of negation to go through. Suppose a proposition $p$ is neither true nor false in some situation $s$. Is $\neg p$ true in that situation? If the answer is 'yes' then the contrapositive of Leibniz's Law will state (relative to a situation) that if it is true that $\mathrm{x}$ has a property and it is either (i) false or (ii) neither true nor false that $y$ has that property then it is not true that $x$ and y are identical. We can call this the weaker interpretation of Leibniz's Law. Victory and Restoration may satisfy the antecedent of this principle in virtue of the property of being indeterminately identical to Restoration. But the consequent is not problematic: the negation, in this sense, of 'Victory = Restoration' is consistent with our claim that 'Victory = Restoration' is neither true nor false in $S_{3}$. So Evans must use a different interpretation of negation.

The obvious alternative is the interpretation that $\neg p$ is true iff $p$ is false (I side with this interpretation in the Appendix). On this view, if $p$ is neither true nor false then $\neg p$ is likewise neither true nor false. This is the Strong Kleene interpretation of negation.

But the difficulty for the Evans argument using a Strong Kleene interpretation is that if we use it consistently then Leibniz's Law states something stronger than we initially 
thought. Leibniz's Law is true in every situation: $\forall x \forall y \forall F[[x=y] \rightarrow[F x \rightarrow F y]]$. According to Strong Kleene truth-tables when the antecedent of a material implication is neither true nor false the formula as a whole can only be true if the consequent is true. Thus Leibniz's Law states that if $\mathrm{x}=\mathrm{y}$ is true or neither true nor false then $\mathrm{x}$ and $\mathrm{y}$ will share all properties. But this is much less plausible than the law we started with. It isn't clear why we should accept it, or therefore its contrapositive. (The contrapositive states that if it is true that $\mathrm{x}$ has a property and it is false that $\mathrm{y}$ does then it is false that $\mathrm{x}$ and $\mathrm{y}$ are identical (all relative to a situation).) The argument has been pressed by strengthening an accepted principle without explicitly saying so.

There are further responses available to this challenge as well. One is worth highlighting. It is a requirement of Evans's argument that Victory have the property 'being indeterminately identical to Restoration' in $S_{3}$. The support for this claim is that it is the case in $S_{3}$ that it is neither true nor false that Victory is identical to Restoration. But we might be suspicious of the property 'being indeterminately identical to Restoration'. Using the stricter sense of negation we have outlined it is hard to describe. For it is not simply the complex property 'not being identical to Restoration and not being not identical to Restoration'; for some $\mathrm{x}$ to have this property would be for it to be false that it was identical to Restoration and true that it was identical to Restoration. So we need a different characterisation of it. If being indeterminately identical to Restoration is not simply a complex property constructed from identity and the logical connectives it isn't clear it follows from it being neither true nor false that Victory $=$ Restoration in $S_{3}$ that Victory has it there.

Given our framework, it actually looks very plausible to assert that the following proposition is not true in $S_{3}:$ 'Victory is indeterminately identical to Restoration'. This proposition seems rather to itself be neither true nor false. For we are unclear about the 
identity of Victory, this is the point! So we don't know what to say about Victory in $S_{3 .}{ }^{36}$ Whether it has the property of being indeterminately identical to something or not is itself unclear. Thus whether there is a property that is both had by Victory and lacked by Restoration in $S_{3}$ remains to be shown.

So if we are consistent in our interpretation of negation and the other logical connectives we have no clear problem here. At the very least, we need to hear more in support of the stronger version of Leibniz's Law and in defence of the property of being indeterminately identical to Restoration.

\section{3 'Only $x$ and $y$ '}

A final potential criticism to consider is one raised against theories that seem to make identity extrinsic. The 'only $x$ and $y$ ' principle, from Noonan (1985), claims that it is only the entity or entities involved that can determine whether an identity statement holds. Noonan defends this claim, which is explicitly rejected by Nozick's closest continuer theory. There is substantial weight to the notion that nothing outside the relevant entity or entities should matter to the truth or falsity of their identity. But, on the face of it, the situationalist is, like Nozick, required to jettison this plausible thesis. I wish to argue that this appearance is mistaken.

The situationalist claims that Victory $=$ Restoration in $S_{1}$ but not in $S_{3}$, so something must have changed between $S_{1}$ and $S_{3}$. Given that $S_{1}$ is part of $S_{3}$, what seems to have changed is something external to Victory and Restoration, violating the 'only $x$ and $y$ ' principle. But this appearance is deceptive; the thing that is different between $S_{1}$ and $S_{3}$ is Victory. It is in virtue of there being extra existential situations for Victory in $S_{1}$ that are not in $S_{3}$ that Victory fails restricted transference and hence the identity true in $S_{1}$ need not be true in $S_{3}$. Victory has 
different properties and relations in $S_{1}$ and $S_{3}$. One of those differences is that the relation of identity holds between it and Restoration in the former situation, but not in the latter. So, on our view identity is intrinsic, in the sense that the relation depends solely on the entity (it only depends on Victory and Restoration whether they are identical). But the entity itself can differ between situations, and so the intrinsic relation can too.

This is obviously unsatisfactory if one believes that propositions expressing identities are invariant in truth-values across situations. If so, the relation either always holds or never holds. The relation cannot hold in some situations but not others. So an entity cannot differ between two situations with respect to this relation. But we can reject the invariance of identity across situations and still maintain that identity is intrinsic. ${ }^{37}$ The criticism of our solution from the 'only $\mathrm{x}$ and $\mathrm{y}$ ' principle is therefore misguided.

I hope, therefore, to have assuaged the worries that arise in connection to the necessity of identity, Leibniz's Law and the 'only $x$ and $y$ ' principle, and in so doing to have shown some of the distinctive advantages of the situationalist account.

\section{Conclusion}

I have presented a solution to the infamous puzzle of the Ship of Theseus. The solution comes with significant background machinery, and requires us to reject the universal transference of propositions across situations. It also faces some challenges from the nature of identity, which I have attempted to rebut. The theory is costly: it requires that a number of notions are spelt out in a particular way. For instance, it requires that truth in general, and the truth-values of identities in particular, are relative to a situation. It further requires 
that propositions can be neither true nor false, including those expressing identities. It requires that Leibniz's Law is treated as a schema. It seems to complicate the necessity of identity, negation and the 'only $x$ and $y$ ' principle. But all of these follow from the central and metaphysically weighty use of situations, which can be partial and incomplete. So, situationalism has high costs. Further work would have to be done to see whether paying these costs for a solution to the Ship of Theseus puzzle is prudent. One factor in this evaluation would be close attention to the benefits of the solution, such as its extension to other persistence puzzles, which space has precluded here. Another would be the comparison with alternative solutions. As this stage, I propose a modest conclusion: the novel solution, and the theory behind it, warrants further investigation. 


\section{Appendix ${ }^{38}$}

In this appendix I will provide some more technical material to flesh out the situation theory that undergirds the solutions given in the body of the paper. It takes the form of a semantics for a fragment of English, including the logical connectives and existential and universal quantifiers.

Ingredients

$S$ - a set, the set of possible situations

$A$ - a set, the set of possible individuals

$\leq-$ a partial ordering on $S \cup A$ such that at least the following condition is satisfied:

(i) For no $s \in S$ is there an $a \in A$ such that $s \leq a$

$P$ - the set of propositions

$\leq$ is intuitively understood to be the parthood relation.

We can now set down the logical properties and relations. Truth and falsity are not defined in terms of some other notions; they stand as a primitives in the theory.

Consistency

A set of propositions $A \subseteq P$ is consistent iff there is a $s \in S$ such that all members of $A$ are true in $s$.

Compatibility 
A proposition $p \in \mathrm{P}$ is compatible with a set of propositions $\mathrm{A} \subseteq P$ iff $A \cup\{p\}$ is consistent.

\section{Logical equivalence}

Two propositions $p$ and $q \in P$ are logically equivalent iff the set of situations $B \subseteq S$ in which $p$ is true and the set of situations $C \subseteq S$ in which $q$ is true are such that $B=C$ and the set of situations $D \subseteq S$ in which $p$ is false and the set of situations $E \subseteq S$ in which $q$ is false are such that $D=E$.

\section{Logical consequence}

A proposition $p \in \mathrm{P}$ follows from a set of propositions $\mathrm{A} \subseteq \mathrm{P}$ iff there is no $s \in S$ such that all the members of $A$ are true in $s$ and $p$ is false in $s$.

\section{Validity}

A proposition $p \in \mathrm{P}$ is valid iff $p$ is not false in any $s \in S$.

These notions provide us with a theoretical handle on the formal relations between situations.

We can now provide the truth-conditions of the logical connectives and quantifiers (at the level of Logical Form). It will not be sufficient for us to give the truth conditions for sentences following a Tarski model; because we have two primitive notions (truth and falsity) we shall also have to give the conditions under which sentences are false. These are given below: 
Negation true

For any variable assignment $g:[\text { not } \alpha]^{g}$ is true in $s \in S$ iff $[\alpha]^{g}$ is false in $s$.

Negation false

For any variable assignment $g:[\text { not } \alpha]^{g}$ is false in $s \in S$ iff $[\alpha]^{g}$ is true in $s$.

\section{Conjunction true}

For any variable assignment $g:[\alpha \text { and } b]^{g}$ is true in a situation $s \in S$ iff $[\alpha]^{g}$ and $[b]^{g}$ are both true in $s$.

Conjunction false

For any variable assignment $g$ : $[\alpha \text { and } B]^{g}$ is false in a situation $s \in S$ iff $[\alpha]^{g}$ or $[B]^{g}$ is false in $s$.

\section{Disjunction true}

For any variable assignment $g:[\alpha \text { or } \beta]^{g}$ is true in $s \in S$ iff $[\alpha]^{g}$ or $[B]^{g}$ is true in $s$.

Disjunction false

For any variable assignment $g:[\alpha \text { or } \beta]^{g}$ is false in $s \in S$ iff $[\alpha]^{g}$ and $[\beta]^{g}$ are false in $s$.

The logical connectives, then, have strong Kleene truth-tables. ${ }^{39}$

We shall now turn to universal and existential quantification. Let's begin with existential quantification:

Existential Quantification true 
For any variable assignment $g:[(\text { There is an } x: \alpha) B]^{g}$ is true in a situation $s \in S$ iff there is a variable assignment $g^{\prime}$ which is just like $g$ except possibly for the value it assigns to $x$ (call such an assignment an " $x$-alternative of $g^{\prime \prime}$ ) such that $[\alpha]^{g^{\prime}}$ is true in $s$ and $[B]^{g^{\prime}}$ is true in $s$.

\section{Existential Quantification false}

For any variable assignment $g:[(\text { There is an } x: \alpha) B]^{g}$ is false in a situation $s \in S$ iff there is no $x$-alternative of $g$ such that $[\alpha]^{g^{\prime}}$ is true in $s$ and $[B]^{g^{\prime}}$ is true in $s$.

Existential quantification always receives a truth-value; it is bivalent. By contrast, our conditions for universal quantification determine that it is not bivalent, as guaranteed by the first clause:

\section{Universal Quantification true}

For any variable assignment $g:[(\text { For all } x: \alpha) b]^{g}$ is true in a situation $s \in S$ iff both i) there is an x-alternative $g^{\prime}$ of $g$ such that $[\alpha]^{g^{\prime}}$ is true in $s$ and ii) for all $x$-alternatives $g^{\prime}$ of $g$ the following holds: If $[\alpha]^{g^{\prime}}$ is true in $s$ then $[\beta]^{g^{\prime}}$ is true in $s$.

\section{Universal Quantification false}

For any variable assignment $g:[(\text { For all } x: \alpha) b]^{g}$ is false in a situation $s \in S$ iff there is an $x-$ alternative $g^{\prime}$ of $g$ such that $[\alpha]^{g^{\prime}}$ is true in $s$ and $[B]^{g^{\prime}}$ is false in $s$.

This brings to a close our sketch of a semantics in terms of situations. It will be helpful to end by briefly commenting on the closure principles that hold for this theory of situations. According to the theory presented, the propositions which are in a situation are closed 
under negation, conjunction, disjunction, existential quantification and universal

quantification. It is important, though, that they are not closed under logical consequence.

We have provided both truth conditions and falsity conditions for our logical connectives

and quantifiers. In doing so, we hope that we have made it clearer how our theory of

situations is to be understood and how it will work.

\section{Notes}

${ }^{1}$ In particular, analogue solutions can be given for cases of fission and fusion, arbitrary undetached parts (Tibbles or Theon/Deon cases), statues and lumps, paradoxes of increase and decrease, the problem of change and, with some extra analysis, the problem of the many. (In fact, the Ship of Theseus case can be seen as an example of asymmetric fission.) These solutions are explored in my book manuscript, Pickup (MS), which also gives much more detail on the theory underpinning the solutions.

${ }^{2}$ I have particularly in mind two sorts of solution that seem similar: (i) those solutions that appeal to indeterminate or temporary identity: see Parsons (2000) and Gallois (1998) as paradigmatic examples; (ii) those solutions that appeal to judgements between candidates, like Nozick's (1981) 'closest continuer' theory of personal identity (Noonan (1985) discusses this in the Ship of Theseus case). I suggest in the next paragraph reasons to think that, in both cases, the resemblance is merely that.

${ }^{3}$ This distinguishes the view both from the indeterminate/temporary identity views and the closest continuer approach. As a further point of contrast with Nozick, the underlying view of how objects persist through time is endurantist, rather than in terms of temporal parts. It might be possible to recast situationalism using temporal parts, or the closest continuer theory using endurantism, however.

${ }^{4}$ Contrast this, for instance, with the proposal in Noonan (1985) according to which indeterminacy enters at the level of denotation. The fact that there is such indeterminacy distinguishes the view from Nozick's, where identity statements are true or false (though their being so may depend on other candidates for identity): in the case of a tie both statements are false (see Nozick (1981) 33-34).

${ }^{5}$ See especially sect. 4.2 for the 'determinately identical' worry and reply.

${ }^{6}$ Plutarch first mentions it in his Life of Theseus and Hobbes refined the example in De Corpore II:11

${ }^{7}$ This might be disputed. Some might think that in the third story Victory is clearly identical to one of the two later ships and clearly distinct from the other. This is sensitive to the precise story that is told: the time that has elapsed from the arrival of Victory, the number and size of the replacement parts and the precise process of replacement and storage all make a difference. I have aimed for a story according to which the motivations for taking Victory to be identical to Restoration and to Reconstruction are equally strong, though different. If you find the motivations significantly different in strength, we can adjust the story to bring these closer together to generate the same worries. At some point, the motivations will be equally strong: take this to be the example under consideration for the rest of the paper. Thanks to an anonymous reviewer for pushing me on this.

${ }^{8}$ This, of course, would need further explanation before becoming a well-defined notion.

${ }^{9}$ Exactly what this priority consists in has not been specified. I take it that what it is to be a possible world is less fundamental than what it is to be a situation, because the former is a conjunction of what it is to be a situation and some maximality constraint. But the conceptual and metaphysical dependence relations would need to be spelt out further (thanks to an anonymous reviewer for highlighting this).

${ }^{10}$ See Kratzer (1989): sect. 3.1. See also Elbourne (2005): sect. 2.2.1, where he endorses the same view. Armstrong's exposition is found towards the end of his Universals and Scientific Realism (1978) Vol. I. 
${ }^{11}$ Thanks to an anonymous reviewer for highlighting the slightly different formulation needed for merely possible situations. Of course, some views of which particulars exist would avoid the need for such a tweak (see, e.g., Williamson (2013) for the view that what I call here 'merely possible' particulars actually exist, and indeed are necessary).

${ }^{12}$ Of course, this is an incomplete account, as we have not specified what particulars and relations are. But I don't see any tension in what follows with any theory of these. It is also worth pointing out that we thus take situations to be compositional: composed of particulars and relations. For us they are not therefore ontologically primitive (though this is a coherent view).

${ }^{13}$ This draws on the Austinian insight that the truth of our utterances depends both on what is said and of what the thing is said. See Austin (1950).

${ }^{14}$ I will take propositional truth to be fundamental, so if any other entities are truth-apt (e.g. statements or utterances), the truth of these entities will be reducible to the truth of certain propositions in certain situations. These other entities might seem to be true simpliciter, but their truth depends on propositional truth in a situation.

There is an interesting question (raised by an anonymous reviewer) about the status of propositions that concern the truth of propositions in situations. For instance, imagine that the proposition ' $p$ is true in $s$ ' is itself true. Given I want to deny that any proposition is true simpliciter, in which situation(s) is this proposition true? I think we have options here. One is to say that it's true in the situation composed of all other situations, the situational top element (though this commits one to the existence of such a situation). Another is that it is true in all extensions of $s$ (though this commits one to universal transference of such propositions, as discussed later). A third is that it is true in all situations whatsoever (though this commits one to such propositions having a very strong form of necessity argued against below). I prefer the first of these options, but any would do.

${ }^{15}$ Austin's way of dividing up the terrain was slightly different, as his propositions essentially refer to situations. For us, propositions are trans-situational; they are not generally restricted to particular situations. Statements or assertions of them may involve particular situations, however.

${ }^{16}$ For example, whether one favours a correspondence or a coherence theory of truth will have an impact on one's interpretation of truth in a situation.

${ }^{17}$ Thus there are some situations in which $[p \wedge \neg p]$ is neither true nor false. However on the definitions given in the Appendix $p$ and $\neg p$ are inconsistent and $\neg[p \wedge \neg p]$ is valid. Similarly, 'Tom is a married bachelor' can be neither true nor false, for example in situations that don't contain Tom. This connects to our later discussion of necessity in section 4.1 .

${ }^{18}$ For the sake of simplicity, I assume that the mereological relations between situations will be classical.

${ }^{19}$ This will have to be tweaked for us, given the situation theory we accept. Parthood will be defined negatively: $s^{\prime} \leq s$ iff $s$ contains all the particulars in $s^{\prime}$ and none of those particulars is not instantiating a property or relation which it does in $s$. With ' $t$ ' indicating a more complicated combination function, we can still assert $s^{\prime} \leq s$ iff $s+s^{\prime}=s$.

${ }^{20}$ There are more esoteric cases which also put pressure on universal transference, including time-travel, transworld identity and propositions which refer indexically to the situation they are in. There is not space to investigate these here.

${ }^{21}$ There are significant connections between situationalism and other views in the philosophy of time. It is indebted to adverbialism and particularly the SOFism of Hanslanger (2003), though it moves away from them in some key respects. One interesting candidate for the ontology and metaphysics that could underlie the denial of universal transference can be drawn from the realist view of tense called Fragmentalism, outlined in Fine (2005). According to this view reality as a whole is not fundamentally coherent, but smaller fragments of reality are. This primacy of smaller fragments of reality would correspond to the primacy of smaller situations in containing truths. Pickup (MS) gives a thorough treatment of these issues, which can only be mentioned here.

${ }^{22}$ By this, I wish to claim not just that there is some representational indeterminacy (which would be akin to Noonan's approach, for example), but that there is real metaphysical indeterminacy here, i.e. reality itself is not settled as to whether Victory is identical to Restoration (and likewise for Reconstruction) in $S_{3}$.

${ }^{23}$ See note 7 for ways the case could be adjusted for those whose intuitions vary.

${ }^{24}$ See Pickup (MS) for details. See also note 21 for some further references.

${ }^{25}$ See Barcan (1947) and Kripe (1971) 
${ }^{26}$ C.f. the definition of validity in the Appendix, which likewise states that a proposition is valid if it is not false in any situation. Vagueness would need to be discussed to give a full defence of this interpretation of necessity.

${ }^{27}$ Kripke's discussion raises but doesn't answer the question of whether $\mathrm{a}=\mathrm{a}$ in a world or situation in which a does not exist. See Kripke (1971) 137. He wants instead to use a weaker notion of identity (though not the one I use here). This question will matter below.

${ }^{28}$ It is worth pointing out that if an identity $p$ is neither true nor false in a situation then $\square p$ is also neither true nor false in that situation. Therefore the T-axiom, which states $\square p \rightarrow p$, holds within every situation. However, the truth of $\square p$ in some situation $s$ will not entail $p$ in some other situation $s^{*}$.

${ }^{29}$ Thanks to an anonymous reviewer for the prompt to consider this.

${ }^{30}$ An unrestricted version of Leibniz's Law would state that if $a=b$ in any situation then they must share all properties in all situations. This goes against the partial ethos of situation theory; what is true in one situation needn't be true in another. The necessity of identity, of course, is relevant here but has been discussed above. I should also specify that the situationalist is able to identify particulars that are in different situations: e.g. she can capture the claim that $a$ in $s$ is identical to $b$ in $s^{\prime}$. She can do so by construing it $a s: a=b$ in the situation composed of $s+s^{\prime}$. The identity of entities in different situations is their identity in the combined situation. Thus even cross-situational identities are identities within a situation, and Leibniz's Law holds of such individuals within the combined situation.

${ }^{31}$ Victory does have the property 'is-identical-to-Reconstruction-in- $S_{2}$ ', but Victory has this in situations other than $S_{1}$. By introducing the complex property, the situation we are considering changes.

32 See Stalnaker (1986) for a similar way of dealing with identity, though across possible worlds rather than situations.

${ }^{33}$ Note that, as mentioned above, this principle holds within situations. The universal quantifiers are therefore restricted to the situation under consideration. Sometimes the principle is formulated with a biconditional [Fx $\leftrightarrow$ Fy], but this doesn't make a material difference here.

${ }^{34} \mathrm{~A}$ formal contradiction can be derived if $\mathrm{x}$ being distinct from some $\mathrm{y}$ entails $\mathrm{x}=\mathrm{y}$ not being indeterminate, which is plausible on standard interpretations of the determinacy operator.

${ }^{35}$ Strictly speaking, Evans's argument requires that the terms 'Victory' and 'Restoration' have precise designations in $S_{3}$, which might be dubious in the former case. This line of response will be passed over.

${ }^{36}$ This might lead us to claim that Victory is indeterminate with respect to all properties in $S_{3}$. While this would guarantee a response to Evans's argument, it is a stronger supposition than we need, so it seems more prudent to hold back from the claim.

${ }^{37}$ One obscuring factor is that such an invariance of identity across situations would entail that identity is intrinsic. For if identity does not vary from situation to situation then the truth-value of a proposition expressing an identity will be fixed across all situations. This means that whether or not the identity holds is independent of the rest of the situation: it is independent of all external factors. However, although invariance of identity entails that identity is intrinsic, the converse is not the case. Identity being intrinsic doesn't say anything about whether it can vary across situations.

${ }^{38}$ The following outline of a situation semantics is particularly indebted to Kratzer (1989) but there are significant theoretical differences.

${ }^{39}$ The conditional is reducible, as with traditional propositional logic, to the other logical connectives. It is true and false in all the same cases as the negation of: [ $\alpha$ and not $B]$.

Acknowledgements I am grateful to a number of individuals for feedback on this paper: Gonzalo

Rodriguez-Pereyra, Nick Jones, Josh Parsons, JD Lafrance, Al Wilson, Denis Robinson, Stephen Williams

and two very helpful anonymous referees for this journal. The publication of this article has been

supported by the Templeton World Charity Foundation, via my participation in The Metaphysics of

Entanglement project based in the Faculty of Philosophy, Oxford University. 


\section{References}

Austin, John L. 1950. "Truth". Aristotelian Society Supp. 24:111-29

Armstrong, David. 1978. Universals and Scientific Realism. Cambridge: Cambridge University Press.

Barcan, Ruth C. 1947. "The Identity of Individuals in a Strict Functional Calculus of Second Order." Journal of Symbolic Logic 12: 12-15.

Barwise, Jon. 1988. The Situation in Logic. Stanford: CSLI.

and John Perry. 1999. Situations and Attitudes. Stanford: CSLI.

Elbourne, Paul. 2005. Situations and Individuals. Cambridge, MA: MIT Press.

Evans, G. 1978, “Can There Be Vague Objects?” Analysis, 38: 208.

Fine, Kit. 2005. "Tense and Reality." In Modality and Tense: Philosophical Papers. Oxford: Oxford University Press, 261-320.

Gallois, Andre. 1998. Occasions of Identity: The metaphysics of Persistence, Change, and Sameness. New York: Oxford University Press.

Haslanger, Sally. 2003. “Persistence Through Time.” In M. Loux and D. Zimmerman (eds.) The Oxford Handbook of Metaphysics. Oxford: Oxford University Press.

Hobbes, Thomas. 1839. De Corpore. In Molesworth (ed.) English Works of Thomas Hobbes, London: J. Bohn Kratzer, Angelika. 1989. "An Investigation of the Lumps of Thought." Linguistics and Philosophy 12: 607-653. Kripke, Saul. 1971. “Identity and Necessity.” In M. K. Munitz (ed.) Identity and Individuation. New York: New York University Press.

Noonan, Harold. 1985. "The Closest Continuer Theory of Identity." Inquiry 28:195-229

Nozick, Robert. 1981. Philosophical Explanations. Oxford: Clarendon Press.

Parsons, Terence. 2000. Indeterminate Identity: Metaphysics and Semantics. Oxford: Oxford University Press. Pickup, Martin. MS. Reality in Pieces: A Theory of How the World Is.

Salmon, Nathan. 1981. Reference and Essence. Princeton: Princeton University Press.

Stalnaker, Robert. 1986. "Counterparts and Identity." Midwest Studies in Philosophy 11: 121-40.

Williamson, Timothy. 2013. Modal Logic as Metaphysics. Oxford: Oxford University Press. 